\title{
CIENCIAMATRIA
}

Revista Interdisciplinaria de Humanidades, Educación, Ciencia y Tecnología

Año VII. Vol. VII. №13. Julio - Diciembre. 2021

Hecho el depósito de ley: pp201602FA4721

ISSN-L: 2542-3029; ISSN: 2610-802X

Universidad Nacional Experimental Francisco de Miranda (UNEFM). Santa Ana de Coro. Venezuela

María Elena Sevilla Rodríguez; Ana Zulema Castro-Salazar

DOI 10.35381/cm.v7i13.478

\section{Padlet como estrategia de enseñanza colaborativa en el proceso de aprendizaje}

Padlet as a collaborative teaching strategy in the learning process

\author{
María Elena Sevilla-Rodríguez \\ maría.sevilla.39@est.ucacue.edu.ec \\ Universidad Católica de Cuenca, El Tambo \\ Ecuador \\ https://orcid.org/0000-0001-5981-6811 \\ Ana Zulema Castro-Salazar \\ azcastros@ucacue.edu.ec \\ Universidad Católica de Cuenca, Azogues \\ Ecuador \\ https://orcid.org/0000-0002-3837-314X
}

Recibido: 10 de marzo de 2021

Aprobado: 15 de junio de 2021 


\author{
CIENCIAMATRIA \\ Revista Interdisciplinaria de Humanidades, Educación, Ciencia y Tecnología \\ Año VII. Vol. VII. N¹3. Julio - Diciembre. 2021 \\ Hecho el depósito de ley: pp201602FA4721 \\ ISSN-L: 2542-3029; ISSN: 2610-802X \\ Universidad Nacional Experimental Francisco de Miranda (UNEFM). Santa Ana de Coro. Venezuela \\ María Elena Sevilla Rodríguez; Ana Zulema Castro-Salazar
}

\title{
RESUMEN
}

La importancia de generar ambientes de trabajo colaborativos y activos en donde se dé un aprendizaje significativo, a través del uso de herramientas tecnológicas para crear clases motivadoras, comunicativas e innovaras. La investigación tiene como objetivo el analizar de qué manera Padlet puede aportar como herramienta de trabajo colaborativo para el proceso de enseñanza, el cual fue verificado mediante la aplicación de una metodología de investigación de tipo descriptivo, no experimental, de cohorte transversal mediante la aplicación de la encuesta compuesta de 11 preguntas, del cual se obtuvieron los datos que fueron analizados a través del programa SPSS, que permitió definir la problemática y a su vez plantear una propuesta, de capacitación docente en cuanto al Uso del Padlet como una estrategia de aprendizaje colaborativa.

Descriptores: Enseñanza asistida por ordenador; aprendizaje en línea; educación inicial. (palabras tomadas del Tesauro UNESCO).

\begin{abstract}
The importance of generating collaborative and active work environments where significant learning takes place, through the use of technological tools to create motivating, communicative and innovative classes. The objective of the research is to analyze how Padlet can contribute as a collaborative work tool for the teaching process, which was verified by applying a descriptive, non-experimental, cross-sectional cohort research methodology through the application of the survey composed of 11 questions, from which the data were obtained that were analyzed through the SPSS program, which allowed defining the problem and in turn raising a proposal for teacher training regarding the Use of the Padlet as a collaborative learning strategy.
\end{abstract}

Descriptors: Computer assisted instruction; electronic learning; initial education. (words taken from the UNESCO Thesaurus). 


\section{CIENCIAMATRIA \\ Revista Interdisciplinaria de Humanidades, Educación, Ciencia y Tecnología \\ Año VII. Vol. VII. №13. Julio - Diciembre. 2021 \\ Hecho el depósito de ley: pp201602FA4721 \\ ISSN-L: 2542-3029; ISSN: 2610-802X \\ Universidad Nacional Experimental Francisco de Miranda (UNEFM). Santa Ana de Coro. Venezuela}

María Elena Sevilla Rodríguez; Ana Zulema Castro-Salazar

\section{INTRODUCCIÓN}

La actual situación de confinamiento, por un problema se salud pública, ha permitido determinar las dificultados que ha tenido el sistema educativo durante este tiempo, el cual generó una serie de problemas que impidió obtener una educación de calidad y calidez.

Es así que los diferentes estudios sobre el tema educativo, han develado muchos problemas pedagógicos, tal es el caso de la investigación desarrollada por Silva (2017), quien señala sobre el tema que, para conseguir un método centrado en el trabajo del estudiante, se requiere de un cambio metodológico, en donde las actividades sean activas y participativas, en concreto se establece que es el docente quien requiere de un cambio de didáctica y pedagogía, para impartir clases, es decir se ve la necesidad de actualizarse y desarrollar competencias digitales para la formación.

Otro de los trabajos investigativos desarrolladas en España es de Pérez-Ortega (2017), quien indica que, hoy en día el uso de las herramientas tecnológicas informáticas dentro del contexto educativo, está todavía en discusión, pues se determina que los docentes y dicentes aún no están preparados para ingresar dentro de este proceso en done se potencia el uso de instrumentos pedagógicos digitales. Lo que implica que tanto los docentes como los estudiantes requieren de una formación en competencias digitales para crear un ambiente educativo didáctico, activo, participativo y colaborativo, a través de la virtualidad.

En el Ecuador según el informe presentado por la Organización de las Naciones Unidades para la Educación, la Ciencia y la Tecnología [UNESCO] (2020), en donde se estipula que con el inicio de la pandemia, el sistema educativo afronto varios problemas referentes a la continuidad de la educación, que generó errores tanto en los establecimientos como en los docentes, quienes no contaron con las estrategias metodológicas que les permitan cumplir con una educación de calidad, sino que por falta de conocimientos en metodologías activas con el uso de las TIC, y la aplicación de herramientas tecnológicas, generaron una educación carente de calidad y conductista. 


\section{CIENCIAMATRIA \\ Revista Interdisciplinaria de Humanidades, Educación, Ciencia y Tecnología \\ Año VII. Vol. VII. N¹3. Julio - Diciembre. 2021 \\ Hecho el depósito de ley: pp201602FA4721 \\ ISSN-L: 2542-3029; ISSN: 2610-802X \\ Universidad Nacional Experimental Francisco de Miranda (UNEFM). Santa Ana de Coro. Venezuela}

María Elena Sevilla Rodríguez; Ana Zulema Castro-Salazar

Para Portillo-Torres (2017), la educación en el país ha cambiado de forma drástica, ya que desde años atrás se han venido manejado concepciones metodológicas pasivas y conductistas, en donde los docentes no han visto la necesidad de actualizarse en forma innovadora en cuanto al uso de las estrategias activas como en las TIC, lo cual es un problema que afecto de forma directa al desarrollo de los aprendizajes significativos de los estudiantes.

Es así, que se plantea esta investigación de innovación tecnológica, en donde se da a conocer la importancia de tener por parte del docente competencias digitales, las cuales le ayudaran a conseguir una educación mixta, presencia y virtual, las dos compactadas para conseguir un fin en común.

Dentro de la investigación se planteó como objetivo el Analizar de qué manera Padlet puede aportar como herramienta de trabajo colaborativo para el proceso de enseñanza. Siendo la misma una implementación de una metodología innovadora que requiere de la aplicación de competencias digitales de parte de los docentes para desarrollar un aprendizaje significativo

Según, Mora-Vicarioli y Hooper-Simpson (2016), exponen que el aprendizaje colaborativo, tiene mucha importancia, cuando este usa de una o varias herramientas digitales, pues permiten la comunicación y la colaboración para la consecución de los objetivos educativos. Por consiguiente, es de mucha importancia que el nuevo modelo educativo incluya en sus actividades las TIC, para fortalecer el aprendizaje de los estudiantes.

Según Giler-Loor et al. (2020), en la investigación planteada sobre el uso del Padlet como herramienta interactiva se pude deducir que este permite estimular las estructuras mentales en el fortalecimiento del aprendizaje, a más mencionan que las herramientas tecnológicas, permiten innovar en la creación de ambientes colaborativos; además, de que se evidencia que estos aumentan y mejoran la experiencia del proceso de aprendizaje, dicha afirmación investigativa, permite validar la investigación y establecer la importancia de realizar la misma a fin de destacar la eficacia de la herramienta de 


\section{CIENCIAMATRIA \\ Revista Interdisciplinaria de Humanidades, Educación, Ciencia y Tecnología \\ Año VII. Vol. VII. N¹3. Julio - Diciembre. 2021 \\ Hecho el depósito de ley: pp201602FA4721 \\ ISSN-L: 2542-3029; ISSN: 2610-802X \\ Universidad Nacional Experimental Francisco de Miranda (UNEFM). Santa Ana de Coro. Venezuela}

María Elena Sevilla Rodríguez; Ana Zulema Castro-Salazar

trabajo colaborativo para el proceso de enseñanza y a su vez conseguir que las clases virtuales sean activas, innovadoras y sobre todas creativas en un ambiente de trabajo colaborativo, dentro de un marco de respeto y lograr un aprendizaje significativo.

\section{DESARROLLO}

La investigación planteada se basa en el uso de herramientas digitales para lograr el aprendizaje colaborativo, el cual permite la consecución de objetivos educativos. Es así que se analizan varios trabajos realizados sobre el tema iniciando con una revisión a nivel internacional donde se reconoce las investigaciones realizadas en España por parte de: Murcia, Tejedor y Lancheros (2017), quienes en su trabajo concluyen que las herramientas digitales tecnológicas han mejorado el proceso de enseñanza aprendizaje, pues se ha visto que se ha pasado de una metodología conductista a una metodología activa - participativa, en donde los estudiantes crean su aprendizaje. Esta investigación es aplicada a estudiantes de 6to grado, en el área de Historia, en donde se implementó herramientas multimedia, que facilitaron la comprensión de la asignatura y por ende una mejora significativa en el aprendizaje de los estudiantes.

En el mismo país, Santos y Coello (2018), en su investigación en donde se busca el aprendizaje de la lengua de dos contextos diferentes como Japón e Islandia, indican que el uso de la herramienta Padlet, permitió el desarrollo de la competencia digital para la adquisición de la lengua meta. Lo cual implico aplicar estrategias metodológicas activas y la plataforma, en donde se obtuvo el desarrollo de capacidades colaborativas entre estudiantes y docentes a fin de dar una consecución de los objetivos y a su vez generar un espacio que promovió la interacción y comunicación.

Para Gacía-Valcárcel (2014), mencionan que las tecnologías digitales brindan a la sociedad nuevas formas de aprender de forma colaborativa, ya que desarrolla capacidades digitales en los estudiantes los ayudan a solucionar dificultades, lo que implica que a través del uso de la tecnología y la aplicación de estrategias colaborativas 


\section{CIENCIAMATRIA \\ Revista Interdisciplinaria de Humanidades, Educación, Ciencia y Tecnología \\ Año VII. Vol. VII. №13. Julio - Diciembre. 2021 \\ Hecho el depósito de ley: pp201602FA4721 \\ ISSN-L: 2542-3029; ISSN: 2610-802X \\ Universidad Nacional Experimental Francisco de Miranda (UNEFM). Santa Ana de Coro. Venezuela}

María Elena Sevilla Rodríguez; Ana Zulema Castro-Salazar

los alumnos tendrán competencias trascendentales que los ayudarán a solucionar problemas cotidianos.

En el caso de las investigaciones realizadas en la parte de América Latina, se reconoce el caso de Pérez-Ortega (2017), que en su investigación realizada en México, establece que el uso de recursos educativos digitales permitió que los estudiantes den gran importancia de los factores sociales y culturales propios de la región, mediante el diálogo y la comprensión de lenguas, situación que se logró a través la implementación de herramientas digitales, que hicieron de la hora clase innovadora, motivadora y sobre todo despertó el interés de los estudiantes por aprender, a su vez se fomenta el trabajo colaborativo, ya que son los alumnos quienes construyen su aprendizaje de forma colaborativa y desarrollan competencias integradoras. .

Otro trabajo a reconocer es el de Mora-Vicarioli y Hooper-Simpson (2016), quienes realizaron la investigación en Costa Rica de la cual se dedujo que el trabajo colaborativo mediante el uso de ambientes virtuales género en los estudiantes la organización, el autoaprendizaje y las habilidades de comunicación para poder conseguir la culminación de proyectos educativos, una vez hecha esta precisión se puede deducir que las herramientas digitales permiten de forma fácil el desarrollo del trabajo colaborativo y a su vez el desarrollo de competencias y destrezas de aprendizaje.

Según García et al. (2018), en su trabajo investigativo concluyen que el uso de las TICS, está ofreciendo a los estudiantes gran posibilidad de adquirir y ampliar sus conocimientos mediante las redes informáticas o internet, con lo que los establecimientos están en la obligación de implementar centros informáticos, plataformas virtuales y TIC, en donde se dé el desarrollo de competencias digitales, que ayuden a aplicar una metodología constructivista en donde el estudiante sea el que construya su aprendizaje y el docente sea quien oriente al alumno para conseguir una educación de calidad.

En lo que respecta a Ecuador, la investigación realizada por Giler-Loor et al. (2020), quien indica que en la actualidad el proceso de enseñanza aprendizaje, están ya inmiscuidos los recursos tecnológicos con los cual es el docente y los estudiantes tienen la facilidad 


\section{CIENCIAMATRIA \\ Revista Interdisciplinaria de Humanidades, Educación, Ciencia y Tecnología \\ Año VII. Vol. VII. N¹3. Julio - Diciembre. 2021 \\ Hecho el depósito de ley: pp201602FA4721 \\ ISSN-L: 2542-3029; ISSN: 2610-802X \\ Universidad Nacional Experimental Francisco de Miranda (UNEFM). Santa Ana de Coro. Venezuela}

María Elena Sevilla Rodríguez; Ana Zulema Castro-Salazar

de compartir ideas, perspectivas y conocimientos a fin de ir adquiriendo un aprendizaje significativo. El hecho de compartir ideas es a través de estrategias colaborativas, las cuales se pueden desarrollar mediante el uso de herramientas digitales o estrategias constructivistas.

De igual forma Espinel A (2020), concuerda en mencionar que las condiciones actuales permiten el uso de las herramientas tecnológicas, ha implicado un cambio de conducta y actuar en los individuos, así como el hecho de que los estudiantes son quienes piden a sus docentes el usar herramientas digitales para hacer la clase más activa y participativa. A su vez se da la implementación del trabajo colaborativo en donde se desarrollan destrezas como la reflexión, análisis, conceptualización, entre otras, que hacen en el estudiante que cambie su forma de pensar.

Mientras que para Zuña, Romero, y Palma (2020), en su investigación relacionada a las plataformas virtuales y su relación con el trabajo colaborativo, indica que tienen un enfoque constructivista, en donde se organiza el trabajo diario de la escuela dentro y fuera del aula, a fin de transformarla en una práctica social, en donde intervienen todos los estudiantes, mediante el uso de plataformas virtuales o herramientas tecnológicas que fomentan la colaboración dentro del hecho educativo.

\section{Teorías que se relacionan con este trabajo investigativo}

Entre las teorías de aprendizaje analizadas se determina que las que se relaciona con las teorías Experiencial y de Aprendizaje Social, que desde los autores Arancibia et al. 2008), quienes plantean que la teoría experiencial fue estructura por Rogers en el año de 1951, de la cual menciona que el ser humano aprende en base a su experiencia y la teoría de Aprendizaje Social propuesta por Bandura en 1969, en donde establece que el individuo modela su conducta según los factores externos que influyen sobre su voluntad. Las dos teorías antes planteadas se relacionan con la temática, en la parte del trabajo del estudiante, pues se reconoce que el alumno adquiere un conocimiento significativo cuando lo experimenta o vive, dicha noción modifica la conducta del educando a fin de 


\section{CIENCIAMATRIA \\ Revista Interdisciplinaria de Humanidades, Educación, Ciencia y Tecnología \\ Año VII. Vol. VII. N¹3. Julio - Diciembre. 2021 \\ Hecho el depósito de ley: pp201602FA4721 \\ ISSN-L: 2542-3029; ISSN: 2610-802X \\ Universidad Nacional Experimental Francisco de Miranda (UNEFM). Santa Ana de Coro. Venezuela \\ María Elena Sevilla Rodríguez; Ana Zulema Castro-Salazar}

conseguir que el mismo este en capacidad de aplicar este conocimiento sea conceptual, procedimental y actitudinal en su vida diaria.

\section{Educación virtual}

Para conceptualizar lo que es la educación virtual, se establece primero lo que es la educación, que para Guadalupe y Zúñiga (2012), lo conceptualiza a la misma como un proceso por el cual las personas asimilan conocimientos, valores, habilidades, destrezas y actitudes que permiten su desarrollo individual y social, a través de la participación integra y efectiva en la construcción de su aprendizaje, sea este de forma individual o social.

Mientras que la educación virtual para Nieto (2012), es la parte escolarizada en donde se da el uso de herramientas digitales, que permiten la interacción entre los individuos y a su vez el desarrollo de competencias cognitivas, sociales y procedimentales, los cuales son básico para un aprendizaje significativo. Este tipo de educación virtual pretende que los miembros de la comunidad educativa, se vuelvan expertos digitales en donde se dé el uso de distintas plataformas y herramientas digitales, que conlleven a un aprendizaje autónomo o colectivo.

Entre los elementos que se usan comúnmente en la educación virtual están las plataformas y herramientas digitales las cuales como se menciona permiten un aprendizaje activo sea este autónomo o colaborativo.

Para Zuña, et al. (2020), las plataformas virtuales, son el cúmulo de herramientas tecnológicas, que admiten el desarrollo de la enseñanza de forma individual y social, a su vez logra la interacción activa entre el profesor y el alumno, siendo el docente el guía y quien apoya de forma constante al estudiante, a su vez es quien elabora recursos pedagógicos mediante el uso de herramientas digitales y las envía para que el estudiante desarrolle su aprendizaje de forma individual o colaborativa. 


\section{CIENCIAMATRIA \\ Revista Interdisciplinaria de Humanidades, Educación, Ciencia y Tecnología \\ Año VII. Vol. VII. N¹3. Julio - Diciembre. 2021 \\ Hecho el depósito de ley: pp201602FA4721 \\ ISSN-L: 2542-3029; ISSN: 2610-802X \\ Universidad Nacional Experimental Francisco de Miranda (UNEFM). Santa Ana de Coro. Venezuela}

María Elena Sevilla Rodríguez; Ana Zulema Castro-Salazar

\section{El Padlet como herramienta digital de aprendizaje}

En el trabajo se destaca la importancia del Padlet en el aprendizaje de los estudiantes, por lo que se conceptualiza esta herramienta digital, que para Viñas Meritxell (2017), menciona que es una herramienta digital de espacio colaborativo en una nube, que permite presentar recursos digitales y mostrar ideas de forma visual. Es decir, se puede considerar como una pizarra digital en donde el docente puede desarrollar su tema de clase, mediante el uso de distintas herramientas digitales para cumplir con los objetivos educativos y desarrollar competencias digitales en los estudiantes.

Entre las características de esta herramienta digital esta que es gratuita y versátil, ya que permite el uso de varias pizarras de forma libre, incentiva la colaboración dentro de un equipo, es decir que activa la comunicación y reflexión entre los estudiantes, a más de ser un espacio para el diálogo, siempre promueve el aprendizaje ya que motiva a los alumnos a trabajar dentro de ella, pues el hecho de ser llamativa e innovadora capta el interés de los que la usan, se puede concluir que es una herramienta que el docente puede usar para conseguir el aprendizaje de sus estudiantes mediante la imaginación, la creatividad y el dialogo entre los estudiantes a través del trabajo colaborativo.

En relación al trabajo docente ¿Qué actividades puede realizar el docente con esta herramienta?, para dar contestación a esta interrogante se analiza la información de Godoy (2020), quien en su artículo menciona que las actividades a realizar por el docente pueden ser: lluvia de ideas sobre un tema, declaración o proyecto, un portafolio estudiantil digital, eventos escolares, mapas mentales, conocimientos previos, discusiones de libros a través de debates, mapas conceptuales, líneas de tiempo, exposiciones pictóricas, narraciones escritas y gráficas, entre otras acciones que ayudan al docente para desarrollar un aprendizaje virtual en cada una de las áreas que trabaje.

Como se menciona anteriormente el uso del Padlet permite el trabajo colaborativo, ya que en su uso permite el involucramiento de los estudiantes y el docente para crear, y aprender de forma colaborativa, al ser este uno de los objetivos de esta herramienta se procede a conceptualizar los que es el trabajo colaborativo en el proceso de enseñanza 


\section{CIENCIAMATRIA \\ Revista Interdisciplinaria de Humanidades, Educación, Ciencia y Tecnología \\ Año VII. Vol. VII. N¹3. Julio - Diciembre. 2021 \\ Hecho el depósito de ley: pp201602FA4721 \\ ISSN-L: 2542-3029; ISSN: 2610-802X \\ Universidad Nacional Experimental Francisco de Miranda (UNEFM). Santa Ana de Coro. Venezuela \\ María Elena Sevilla Rodríguez; Ana Zulema Castro-Salazar}

aprendizaje. Y se analiza el concepto Revelo et al. (2018), quienes su artículo lo definen como un proceso en el que el estudiante aprende más de lo que aprendería solo o de forma autónoma, por lo que se ve la importancia de trabajar en grupo para conseguir los objetivos propuestos de forma más fácil y cognitiva.

\section{Trabajo colaborativo}

En el mismo artículo se da la importancia al uso de las Técnicas de Aprendizaje Colaborativo (TAC), como formas comunes de ir estructurando un proceso de aprendizaje, para que sea desarrollo por los estudiantes de forma colaborativa En lo que respecta al trabajo colaborativo Lizcano-Dallos et al. (2019), indica que la virtualidad permite al docente integrar la parte procedimental con la tecnología, consiguiendo con ellos acciones de trabajo grupal de los estudiantes o en forma colaborativa, a su vez menciona que dicho trabajo desemboca en un aprendizaje colaborativo a través del uso de las tecnologías y la inclusión en el aprendizaje de los estudiantes.

Lo que para Md Deni y Zainal (2018), indican que uno de los beneficios de las herramientas digitales en la educación, es que permite los docentes pasen de una metodología conductista a una constructivista, en donde el niño a través de estrategias activas colaborativas, consiguen crear un aprendizaje significativo.

\section{METODOLOGÍA}

Se puede mencionar que según Henriquez y Zepeda (2004), la metodología de la investigación es la parte del proceso en donde se integran los conocimientos con la práctica profesional, lo que implica establecer que son los resultados o hallazgo de la investigación en la parte práctica.

Es así que esta investigación es de tipo descriptiva no experimental, que solo se observo a la población para determinar el problema a analizar. Tiene un paradigma de tipo 


\section{CIENCIAMATRIA}

Revista Interdisciplinaria de Humanidades, Educación, Ciencia y Tecnología

Año VII. Vol. VII. N¹3. Julio - Diciembre. 2021

Hecho el depósito de ley: pp201602FA4721

ISSN-L: 2542-3029; ISSN: 2610-802X

Universidad Nacional Experimental Francisco de Miranda (UNEFM). Santa Ana de Coro. Venezuela

María Elena Sevilla Rodríguez; Ana Zulema Castro-Salazar

cuantitativo, ya que se obtuvieron datos de la población, y de un cohorte transversal, puesto que solo se realizo la aplicación del instrumento en un solo tiempo.

En cuanto a la población a estudiar fueron los docentes de la institución, a quienes se les aplicó una encuesta de 11 preguntas referentes al uso del Padlet como una estrategias de trabajo colborativo, dicho instrumento cumplió la fiabilidad mediante la aplicación del método de alfa de Cronbach, con un valor de 0,836, también se puedo identificar que 10 preguntas fueron paramétricas y una no paramétrica. Para la tabulación de los datos se aplico el programa SPSS, en cual se determinó los porcentajes y la comparación entre preguntas, según las resultados obtenidos se puedo determinar la propuesta a la problemática planteada.

\section{RESULTADOS}

Ya aplicada las encuestas y analizados los datos se obtubieron los siguientes resultados.

\section{Tabla 1.}

Metodología Constructivista.

\begin{tabular}{|c|c|c|c|c|c|}
\hline & & Frecuencia & Porcentaje & $\begin{array}{c}\text { Porcentaje } \\
\text { válido } \\
\end{array}$ & $\begin{array}{l}\text { Porcentaje } \\
\text { acumulado }\end{array}$ \\
\hline \multirow[t]{5}{*}{ Válidos } & Siempre & 10 & 35,7 & 35,7 & 35,7 \\
\hline & Casi siempre & 9 & 32,1 & 32,1 & 67,9 \\
\hline & Frecuentemente & 7 & 25,0 & 25,0 & 92,9 \\
\hline & A veces & 2 & 7,1 & 7,1 & 100,0 \\
\hline & Total & 28 & 100,0 & 100,0 & \\
\hline
\end{tabular}

Fuente: Encuesta.

Según la tabla 1, se puede determinar que el 92,9\% de los docentes, aplican frecuentemente una metodología constructivista. Lo que implica determinar que el uso de esta metodología no es común o se la realiza siempre en horas clases, ya que los docentes común mente desarrollan clases expositivas, en donde aplican videos de los 


\section{CIENCIAMATRIA}

Revista Interdisciplinaria de Humanidades, Educación, Ciencia y Tecnología

Año VII. Vol. VII. №13. Julio - Diciembre. 2021

Hecho el depósito de ley: pp201602FA4721 ISSN-L: 2542-3029; ISSN: 2610-802X

Universidad Nacional Experimental Francisco de Miranda (UNEFM). Santa Ana de Coro. Venezuela

María Elena Sevilla Rodríguez; Ana Zulema Castro-Salazar

temas, así como la lectura de los textos que tienen los estudiantes llevando a los mismos a actividades conductistas.

Tabla 2.

Herramientas digitales.

\begin{tabular}{llrrrr}
\hline & & & Porcentaje & Porcentaje \\
& & Frecuencia & Porcentaje & \multicolumn{1}{c}{ válido } & acumulado \\
\hline \multirow{2}{*}{ Válidos } & Siempre & 10 & 35,7 & 35,7 & 35,7 \\
& Casi siempre & 6 & 21,4 & 21,4 & 57,1 \\
& Frecuentemente & 3 & 10,7 & 10,7 & 67,9 \\
& A veces & 6 & 21,4 & 21,4 & 89,3 \\
& Nunca & 3 & 10,7 & 10,7 & 100,0 \\
\cline { 2 - 6 } & Total & 28 & 100,0 & 100,0 & \\
\hline
\end{tabular}

Fuente: Encuesta.

En la tabla 2, del las herramientas digitales el $89,3 \%$ mencionan que los usan a veces para dar clases. Esta situación permite establecer que no hay un uso de herramientas digitales, entre ellas el padlet, lo cual implica que haya una metodologia tradicional, y no una cosntructivista, esta situación se presenta a la falta de conocimiento en cuanto a la aplicación, beneficios y ventajas de usar las herramientas digitales en el proceso de enseñanza aprendizaje. 


\section{CIENCIAMATRIA}

Revista Interdisciplinaria de Humanidades, Educación, Ciencia y Tecnología

Año VII. Vol. VII. №13. Julio - Diciembre. 2021

Hecho el depósito de ley: pp201602FA4721 ISSN-L: 2542-3029; ISSN: 2610-802X

Universidad Nacional Experimental Francisco de Miranda (UNEFM). Santa Ana de Coro. Venezuela

María Elena Sevilla Rodríguez; Ana Zulema Castro-Salazar

\section{Tabla 3.}

Trabajo colaborativo.

\begin{tabular}{llrrrr}
\hline & & & Porcentaje & Porcentaje \\
válido & acumulado \\
\hline \multirow{2}{*}{ Válidos } & Siempre & 10 & 35,7 & 35,7 & 35,7 \\
& Casi siempre & 7 & 25,0 & 25,0 & 60,7 \\
& Frecuentemente & 4 & 14,3 & 14,3 & 75,0 \\
& A veces & 5 & 17,9 & 17,9 & 92,9 \\
& Nunca & 2 & 7,1 & 7,1 & 100,0 \\
\cline { 2 - 6 } & Total & 28 & 100,0 & 100,0 & \\
\hline
\end{tabular}

Fuente: Encuesta.

De acuerdo a la tabla 3, se determina que el $92.9 \%$ de los docentes indican que a veces desarrollan el trabajo colaborativo entre los estudiantes, estableciéndose que la mayoría de las actividades ejecutadas por los niños son individuales esto debido a que son clases virtuales. Y según los maestros es difícil hacer que ellos trabajen en grupo o de forma colaborativa, de allí la necesidad de que los docentes puedan mediante la virtualidad generar actividades grupales o colaborativas, esto a través de acciones que vinculen la tecnología con la pedagogía. 


\section{CIENCIAMATRIA}

Revista Interdisciplinaria de Humanidades, Educación, Ciencia y Tecnología

Año VII. Vol. VII. №13. Julio - Diciembre. 2021

Hecho el depósito de ley: pp201602FA4721 ISSN-L: 2542-3029; ISSN: 2610-802X

Universidad Nacional Experimental Francisco de Miranda (UNEFM). Santa Ana de Coro. Venezuela

María Elena Sevilla Rodríguez; Ana Zulema Castro-Salazar

Tabla 4.

Capacitación en Padlet.

\begin{tabular}{|c|c|c|c|c|c|}
\hline & & Frecuencia & Porcentaje & $\begin{array}{c}\text { Porcentaje } \\
\text { válido }\end{array}$ & $\begin{array}{l}\text { Porcentaje } \\
\text { acumulado }\end{array}$ \\
\hline \multirow[t]{6}{*}{ Válidos } & Siempre & 16 & 57,1 & 57,1 & 57,1 \\
\hline & Casi siempre & 5 & 17,9 & 17,9 & 75,0 \\
\hline & Frecuentemente & 4 & 14,3 & 14,3 & 89,3 \\
\hline & A veces & 2 & 7,1 & 7,1 & 96,4 \\
\hline & Nunca & 1 & 3,6 & 3,6 & 100,0 \\
\hline & Total & 28 & 100,0 & 100,0 & \\
\hline
\end{tabular}

Fuente: Encuesta.

En cuanto a la tabla 4, se evidencia que el $57,1 \%$ de los docentes están de acuerdo en asistir a capacitaciones en donde se pueda mejorar su pedagogía y metodología, ya que se puede observar que los docentes siempre esta auto capacitándose en cuanto a temáticas pedagógicas, pero no lo han hecho en cuanto al uso de herramientas tecnológicas, pues indican que para esas actividades deben de ser de forma presencial y así poder mejorar su metodología y poder desarrollar un aprendizaje colaborativo y significativo. 


\section{CIENCIAMATRIA}

Revista Interdisciplinaria de Humanidades, Educación, Ciencia y Tecnología

Año VII. Vol. VII. N¹3. Julio - Diciembre. 2021

Hecho el depósito de ley: pp201602FA4721

ISSN-L: 2542-3029; ISSN: 2610-802X

Universidad Nacional Experimental Francisco de Miranda (UNEFM). Santa Ana de Coro. Venezuela

María Elena Sevilla Rodríguez; Ana Zulema Castro-Salazar

Tabla 5.

Análisis de Chi-cuandrado de Pearson Tabla de Trabajo colaborativo y Recursos tecnológicos.

\begin{tabular}{|c|c|c|c|c|c|c|c|}
\hline & & & & Recursos tecnoló & icos & & \\
\hline & & Nunca & $\begin{array}{c}\text { A } \\
\text { veces }\end{array}$ & Frecuentemente & $\begin{array}{c}\text { Casi } \\
\text { siempre }\end{array}$ & Siempre & Total \\
\hline Trabajo & Siempre & 0 & 0 & 1 & & 7 & 10 \\
\hline colaborativo & Casi siempre & 0 & 0 & 2 & & 2 & 7 \\
\hline & Frecuentemente & 0 & 1 & 1 & & 2 & 4 \\
\hline & A veces & 0 & 0 & 2 & & 1 & 5 \\
\hline & Nunca & 1 & 0 & 0 & & 0 & 2 \\
\hline Total & & 1 & 1 & 6 & & 12 & 28 \\
\hline & & Prueb & as de $\mathrm{cl}$ & hi-cuadrado & & & \\
\hline & & & Valo & gl & Sig. & asintótica (k & lateral) \\
\hline Chi-cuadradc & Pearson & & & $27,050^{\mathrm{a}}$ & 16 & & 0,041 \\
\hline Razón de vel & ilitudes & & & 19,412 & 16 & & 0,248 \\
\hline Asociación lir & por lineal & & & 6,068 & 1 & & 0,014 \\
\hline $\mathrm{N}$ de casos $\mathrm{v}$ & & & & 28 & & & \\
\hline
\end{tabular}

Fuente: Encuesta.

En la Tabla 5 se relacionan las dos variables de analisis; de modo que, mediante la prueba Chi-cuadrado de Pearson se puede evidenciar el nivel de significación bilateral $\mathrm{P}<0,5$ asumiendo la hipotesis afirmativa $(\mathrm{H} 0)$ que los docentes usan herramientas digitales colaborativas en el proceso de aprendizaje es nula. Por lo tanto las dos variables estan asociadas en un 0,041 de significancia, lo que implica la necesidad de una capaaitación en la formación para el uso de herramientas digitales para desarrollar el aprendizaje colaborativo. 


\section{CIENCIAMATRIA}

Revista Interdisciplinaria de Humanidades, Educación, Ciencia y Tecnología

Año VII. Vol. VII. N¹3. Julio - Diciembre. 2021

Hecho el depósito de ley: pp201602FA4721

ISSN-L: 2542-3029; ISSN: 2610-802X

Universidad Nacional Experimental Francisco de Miranda (UNEFM). Santa Ana de Coro. Venezuela

María Elena Sevilla Rodríguez; Ana Zulema Castro-Salazar

\section{PROPUESTA}

Determinad ya la problemática, se plantea una solución a la misma, la cual tiende una capacitación docente en el uso del Padlet como herramienta digital de trabajo colaborativo para así aplicar una metodología innovadora constructivista y así desarrollar un aprendizaje significativo.

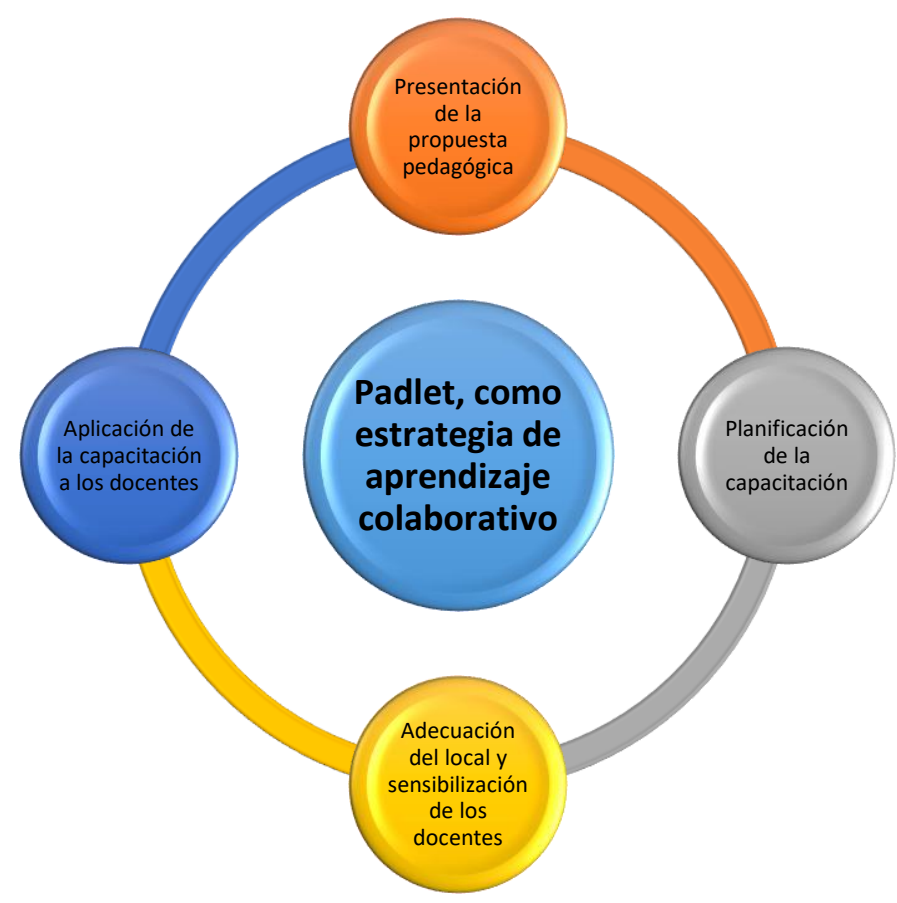

Figura 1. Padlet como estrategia de aprendizaje colaborativo. Elaboración: Los autores.

Se plantea las siguientes actividades para la capacitación docente en el uso del Padlet como estrategia de aprendizaje colaborativa. Para esta propuesta se plantean las siguientes actividades:

Presentación de la propuesta pedagógica a las autoridades de la institución a fin de autorizar la aplicación de la misma dentro del establecimiento educativa. Dicha actividad será mediante la presentación de los resultados diagnósticos de la investigación aplicada a los docentes del cual se determinó que los docentes no manejan herramientas digitales 
Revista Interdisciplinaria de Humanidades, Educación, Ciencia y Tecnología

Año VII. Vol. VII. No13. Julio - Diciembre. 2021

Hecho el depósito de ley: pp201602FA4721

ISSN-L: 2542-3029; ISSN: 2610-802X

Universidad Nacional Experimental Francisco de Miranda (UNEFM). Santa Ana de Coro. Venezuela

María Elena Sevilla Rodríguez; Ana Zulema Castro-Salazar

para desarrollar el trabajo colaborativo y así dar validad a la propuesta de una capacitación.

Planificación de la capacitación docente: lo cual implica formular los objetivos de capacitación, organizar las actividades, los recursos y sobre todo elaborar el manual de uso del Padlet como estrategia de aprendizaje colaborativo.

Adecuación del local y sensibilización de los docentes: en cuanto a la adecuación del local se utilizará el aula o laboratorio de informática con todo lo necesario para que los docentes puedan tener acceso al internet y puedan desarrollar actividades en línea. Se pretende motivar a los docentes a ser parte de esta capacitación innovadora para que ellos puedan aplicar una metodología constructivista e innovadora.

En lo que respecta a la aplicación de la capacitación se los realizará mediante un manual de uso de Padlet en se tendrá paso a paso como crear, desarrollar y aplicar esta herramienta como estrategia de aprendizaje colaborativo.

\section{CONCLUSIONES}

El uso de herramientas digitales permite integrar la pedagogia con la tecnologia, lo que implica que los docentes tengan que capacitarse en cuanto a la temática, para poder aplicar una metodología constructivista.

Se pudo aplicar una metodología descriptiva, de tipo no experimental, con carcater cuantitativo, que permitio recolectar la información mediante una encuesta a la población total de los docentes de la intotución, de quienes se obtuvo datos que permitieron definir la problemática y a su vez una propuesta de solución.

Que la mayoria de docentes aplican común mente una metodología tradicional, durante las clases virtuales, ya que son expositivas, usan videos de youtube y la lectura del texto, 


\section{CIENCIAMATRIA \\ Revista Interdisciplinaria de Humanidades, Educación, Ciencia y Tecnología \\ Año VII. Vol. VII. N¹3. Julio - Diciembre. 2021 \\ Hecho el depósito de ley: pp201602FA4721 \\ ISSN-L: 2542-3029; ISSN: 2610-802X \\ Universidad Nacional Experimental Francisco de Miranda (UNEFM). Santa Ana de Coro. Venezuela}

María Elena Sevilla Rodríguez; Ana Zulema Castro-Salazar

ocasionando con ello el canzancio de los estudiantes, la desmotivación pero sobre todo la falta de comprensión del tema.

Existe un desconocimiento en cuanto al uso de herramientas digitales en las clases sincrónica y asincrónica, pues no saben cuales son y como se las aplica en los proceso de enseñanza.

En cuanto al Padlet, la mayoria desconoce de esta herramienta, en cuanto a su aplicación, uso y desarrollo durante un proceso de aprendizaje, de allí la importancia de capacitar sobre la misma y puedan desarollar clases motivadoras, llamativas, innovadoras y colaborativas

Común mente las actividades de los estudiantes son individuales y no colaborativas, ya que señalan que no pueden desarrollar este tipo de actividades, ya que no saben como hacerlo, situación que se sucita a falta del uso de herramientas digitales innovadoras.

Determinado la problemática y con el objeto de desarrollar clases constructivistas, motivadoras y de trabajo colaborativo se propone la capcitación en el Uso de la Herramienta Padlet, que es uno los recursos virtuales que ayuda mucho en el tema y se puede aplicar de forma fácil y práctica por parte del docente y estudiante.

\section{REFERENCIAS}

Arancibia, V., Herrera, P., \& Strasser, K. (2008). Educacional Psicología [Manual de Psicología Educativa]. https://n9.cl/0tsqg

Edgar Zuña, Wilsón Romero, Julio Palma, C. S. (2020). Aprendizaje colaborativo.pdf [Collaborative learning] (pp. 1-24). https://n9.cl/hkeu57

Espinel Armas, E. E. (2020). La tecnología en el aprendizaje del estudiantado de la Facultad de Ciencias Químicas, Universidad Central del Ecuador. Actualidades Investigativas En Educación, 20(2), 1-39. https://doi.org/10.15517/aie.v20i2.41653

Gacía-Valcárcel, Verónica, C. S. (2014). Las TIC en el aprendizaje colaborativo en el aula de Primaria y Secundaria [ ICT in collaborative learning in the Primary and Secondary classroom]. Comunicar, XXI(42), 65-74. https://n9.cl/4x9k 


\section{CIENCIAMATRIA \\ Revista Interdisciplinaria de Humanidades, Educación, Ciencia y Tecnología \\ Año VII. Vol. VII. N¹3. Julio - Diciembre. 2021 \\ Hecho el depósito de ley: pp201602FA4721 \\ ISSN-L: 2542-3029; ISSN: 2610-802X \\ Universidad Nacional Experimental Francisco de Miranda (UNEFM). Santa Ana de Coro. Venezuela}

María Elena Sevilla Rodríguez; Ana Zulema Castro-Salazar

García Sánchez, M. del R., Reyes Añorve, J., \& Godínez Alarcón, G. (2018). Las Tic en la educación superior, innovaciones y retos. [The ICT in higher education, innovations and challenges]. RICSH Revista Iberoamericana de Las Ciencias Sociales y Humanísticas, 6(12), 299-316. https://doi.org/10.23913/ricsh.v6i12.135

Giler-Loor, D. J., Mendoza, G. K. Z., Saldarriaga, A. M. V., \& Moreira, M. T. V. (2020). Padlet como herramienta interactiva para estimular las estructuras mentales en el fortalecimiento del aprendizaje [Padlet as an interactive tool to stimulate mental structures in strengthening learning]. Dialnet.Unirioja.Es, 6, 1332-1351. https://n9.cl/6t5su

Godoy Carlos. (2020). Antes de utilizar Padlet lee esto - Docente Curador [Before using Padlet read this - Curator Teacher]. https://n9.cl/4rc8s

Guadalupe, M., \& Zúñiga, M. (2012). Revista Iberoamericana para la Investigación y el Desarrollo Educativo [Virtual education as a tool in educational guidance]. 3(5). https://n9.cl/cz2pn

Henriquez Fierro, E., \& Zepeda González, M. I. (2004). Elaboración de un Artículo Científico De Investigación [Preparation of a Scientific Research Article]. Ciencia y Enfermería, 10(1), 17-21. https://doi.org/10.4067/s0717-95532004000100003

Lizcano-Dallos, A. R., Barbosa-Chacón, J. W., \& Villamizar-Escobar, J. D. (2019). ICTaided collaborative learning: Concept, methodology and resources [Aprendizaje colaborativo asistido por TIC: concepto, metodología y recursos]. Magis, 12(24), 524. https://doi.org/10.11144/Javeriana.m12-24.acat

Md Deni, A. R., \& Zainal, Z. I. (2018). Padlet as an educational tool: Pedagogical considerations and lessons learnt [Padlet como herramienta educativa: consideraciones pedagógicas y lecciones aprendidas]. ACM International Conference Proceeding Series, December, 156-162. https://doi.org/10.1145/3290511.3290512

Mora-Vicarioli, F., \& Hooper-Simpson, C. (2016). Trabajo colaborativo en ambientes virtuales de aprendizaje: Algunas reflexiones y perspectivas estudiantiles [Collaborative work in virtual learning environments: Some reflections and student perspectives]. Revista Electrónica Educare., 20(2), 1-26. https://n9.cl/b7kx7 


\section{CIENCIAMATRIA \\ Revista Interdisciplinaria de Humanidades, Educación, Ciencia y Tecnología \\ Año VII. Vol. VII. N¹3. Julio - Diciembre. 2021 \\ Hecho el depósito de ley: pp201602FA4721 \\ ISSN-L: 2542-3029; ISSN: 2610-802X \\ Universidad Nacional Experimental Francisco de Miranda (UNEFM). Santa Ana de Coro. Venezuela}

María Elena Sevilla Rodríguez; Ana Zulema Castro-Salazar

Murcia Castellanos, Yenny Constanza; Tejedor Estupiñan, María Lucia; Lancheros Cuesta, D. Y. (2017). Impacto de una herramienta multimedial en el proceso de Enseñanza-Aprendizaje de la historia en el aula [Impact of a multimedia tool in the process of learning history in the classroom]. 211-229. https://n9.cl/fm4u

Nieto Göller, R. (2012). Educación Virtual O Virtualidad [Virtual Education OR Virtuality]. Historia de La Educación Latinoamericana, 14(19), 137-150. https://n9.cl/qota3

Pérez-Ortega, I. (2017). Creación de Recursos Educativos Digitales: Reflexiones sobre innovación educativa con TIC [Creation of Digital Educational Resources: Reflections on educational innovation with ICT]. International Journal of Sociology of Education, 6(2), 244. https://doi.org/10.17583/rise.2017.2544

Portillo-Torres, M. C. (2017). Educación por habilidades: Perspectivas y retos para el sistema educativo [Skills education: Perspectives and challenges for the education system]. Revista Educación, 41(2), 1. https://doi.org/10.15517/revedu.v41i2.21719

Revelo, O., Collazos, C., \& Jiménez, J. (2018). El trabajo colaborativo como estrategia didáctica para la enseñanza/aprendizaje de la programación: una revisión sistemática de la literatura [Collaborative work as a didactic strategy for teaching / learning programming: a systematic review of the litera. TecnoLógicas, 21(41), 1237799. https://n9.cl/xal5d

Santos, M., \& Coello, C. (2018). Uso de herramientas digitales para la escritura colaborativa en línea: El caso de Padlet [Use of digital tools for collaborative writing online: The case of Padlet]. November, 1-17. https://n9.cl/srr3h

Silva, J. (2017). Un modelo pedagógico virtual centrado en las E-actividades [A virtual pedagogical model focused on E-activities]. Revista de Educación a Distancia (RED), 53. https://doi.org/10.6018/red/53/10

Viñas Meritxell. (2017). Padlet: Configurar una pizarra colaborativa en minutos / Recursos TIC para profesores [Padlet: Set Up a Collaborative Whiteboard in Minutes / ICT resources for teachers]. Revista Académica. https://n9.cl/cesbf 